\title{
Performance Tests for Wireless Real-time Localization Systems to Improve Mobile Robot Navigation in Various Indoor Environments
}

\author{
Yong K. Cho*, Jong-Hoon Youn** and Nam Pham** \\ ${ }^{*}$ Construction Engineering \& Management Division, School of Architectural Engineering \\ and Construction, University of Nebraska-Lincoln, \\ *Computer Science Department, University of Nebraska-Omaha,
}

USA

\section{Introduction}

This research introduces a research effort at the Peter Kiewit Institute in Omaha, Nebraska by investigating the performances of current wireless real-time localization technologies. Futhermore, the research shows how localization technologies can be applied to sensoraided intelligent mobile robots for high-level navigation functions for indoor construction security and material delivery.

Sensor-based exploration enables a robot to explore an environment and to build a map of the explored environment. A critical component of sensor-based exploration is robot's ability to ascertain its location in a partially explored map or to determine that it has entered a new territory. Theoretically, one can determine the $(x, y)$ coordinates of the robot using dead-reckoning - a process that determines the robot's location by integrating data from wheel encoders that count the number of wheel rotations. However, dead-reckoning often fails to accurately position the robot for many reasons, including differential of wheel rotation rate and wheel slippage. Especially when the robot slips, the wheel rotation does not correspond to its movement, and thus encoder data, which reflects the state of the wheel rotation, does not reflect the robot's net motion, thereby causing positioning errors. A global positioning system (GPS) offers an alternative to dead-reckoning, but it is limited to outdoor applications.

Tracking mobile assets in indoor environments is a challenging task, especially for large open spaces such as airport terminals and museums. Among the emerging technologies, mobile devices and wireless technologies are widely recognized as solutions for identifying locations of mobile assets in such areas. However, the integration of these technologies into indoor building space has been limited. For example, one type of building space in which the integration has been particularly slow is a highly congested area with room partitions, metal structures, furnitures, and high traffic of people. Location tracking in such environment often has low valuation attributes, including reliability, security, and performance. In turn, the lack of these attributes has prevented high performance wireless networks from replacing traditional IT systems in critical applications. Critical applications 
where the wireless networking technology can be effectively applied are military training, mobile resource tracking in construction, fire fighter tracking, rare or endangered animal tracking in a natural habitat. Another useful implementation is in hospitals and health care facilities, where a number of problems can be solved effectively and efficiently by taking advantages of the wireless networking technology. A related study estimated that as many as 98,000 people die in the U.S. hospitals each year due to medical errors (Kohn et al., 2000). Using wireless networking technologies to efficiently manage medical records and important assets, these errors and the number of misdiagnoses can be significantly reduced.

Although the wireless networks are identified as the most promising technology to track indoor mobile assets, building environment factors such as building type, business type, and geographical location significantly affect the position accuracy. This paper presents a framework for strategic planning in tracking mobile assets in various indoor environments and scenarios, using three most popular wireless technologies. Then, the paper demonstrates one of the wireless technologies and its integration to mobile robot's path planning system to improve its navigation in an indoor environment. The rest of this paper is structured as follows. Section 2 surveys related works. Section 3 discusses the development and deployment of three wireless sensor technologies for real-time asset tracking in building environments. Also, this section presents experimental results of each deployed tracking system. Section 4 details how an ultra-wide band wireless tracking system can be installed on a robot in order to improve robot's nagivation. Concluding remarks are presented in Section 5 .

\section{Related works}

A number of indoor positioning systems are available in the literature. Among these systems, common positioning techniques include trilateration, multilateration, and location learning.

- Trilateration uses range estimates of the distances between devices and calculates positions of target devices using geometric identities and known locations of other devices. Distances can be estimated with time of arrival (TOA) or signal strength. With TOA, two devices must be synchronized, and messages between the devices are timestamped upon sending and receiving in order to calculate propagation delay. The known propagation delays of signals in a particular medium allow the devices to estimate distance.

- Mutlilateration uses time difference of arrival (TDOA) estimates in which several reference devices measure the difference in arrival times of signals from the target devices. Round-trip time can be used when synchronization is not possible. For triangulation, the angle of arrival (AOA) of a signal is measured using several antennas, and then geometric identities are used for estimating position.

- Location learning makes no range or angle measurements. Instead, the method merely correlates the properties of newly received signals with data available on previously observed signals at known locations.

These basic techniques can be used with a variety of signal types in wireless systems: wireless local area networks (WLANs), wireless sensor networks (WSNs), ultra-wide band (UWB) networks, and RFID systems. 


\section{A. Wireless LAN Based Systems}

The use of radio frequency (RF) properties for determining position in building environments is always challenging because of obstacles such as walls, furnitures, people traffic, and interferences with other RF noises. For this reason, localization in WLANs often relies on learning techniques. One WLAN based tracking system is RADAR (Radio Detection and Ranging) - an indoor tracking system developed at Microsoft Research (Bahl \& Padmanabhan, 2000). RADAR is a learning-based approach which takes advantages of the existing WLAN infrastructures. Localization or tracking with RADAR consists of two phases: a reference signature collection phase and an online estimation phase. During the signature collection phase, a user with a laptop clicks his or her perceived location on a map interface and records the signal strength from all access points (APs) within range. After collecting a sufficiently large database of reference signals, location can then be estimated in the online phase by taking the geographic centroid of the locations of the $k$-nearest (in terms of signal-strength space) reference signatures. The same process may be used with other traditional machine learning algorithms and has been studied on Artificial Neural Networks (Battiti et al., 2002), Bayesian techniques, and Markov models (Haeberlen et al., 2004; Ladd et al., 2002). Variants of the RADAR system are available from commercial vendors such as PanGo (Pango, 2008) and Ekahau (Ekahau, 2008).

\section{B. Wireless Sensor Based Systems}

As one of the earliest location tracking systems for sensor networks, the Active Badge system was developed by AT\&T Cambridge for indoor location tracking using diffuse infrared technology (Want et al., 1992). After Active Badge, AT\&T researchers developed the Active Bat system to improve Active Badge's limitations on 3D location and orientation information (Harter et al., 1999). However, Active Bat requires a large scale of expensive wiring infrastructure to relay information collected by the receivers. To compete with the Active Bat system, Networks and Mobile System research group at the Massachusetts Institute of Technology (MIT) developed the Cricket location-support system which allows applications running on user devices to learn their physical location (Priyantha et al. 2000). The Cricket system consists of beacons (mounted on a wall or ceiling) to emit radio frequency signals and receivers (attached to the user's mobile device) to receive beacons' RF and ultrasonic signals. Unlike the Active Bat system, Cricket does not require a grid of fixed ceiling sensors because the receivers perform the timing and computation function (Skibniewski et al. 2007). However, timing and processing both the ultrasound pulses and RF data may increase computation and power burden on the mobile receivers, and it is not easy to monitor the performance of receivers due to decentralized management (Hightower et al. 2001). MoteTrack is a sensor-based system which runs on 802.15 .4 based motes but uses a process based on RADAR. With MoteTrack, the environment must be equipped with several fixed sensor motes as the existing LAN infrastructure cannot be used. Reference signature collection and the online estimation operate as in RADAR, but MoteTrack has been altered to run in a distributed manner. MoteTrack has the advantage of being entirely based on RF signals and needs relatively fewer beacon nodes to cover a large area of a building, even an area with many obstacles. However, it also has a disadvantage that it requires significantly more configuration prior to deployment. 


\section{Ultra-wideband (UWB) Systems}

Gezici et al. discussed many of the positioning techniques described earlier in the context of UWB systems in which high bandwidths offer potentially high ranging accuracy. They note that the antenna arrays required for the angle of arrival(AOA) make it unsuitable for UWB, but consider ranging with time-based measurements and signal strength measurements. They found that the best results can be obtained with hybrid schemes employing TDOA and TOA both with signal strength measurements (Gezici et al., 2005). Young et al. noted that the high bandwidth of UWB systems allow for high time resolution leading to a natural advantage with TDOA localization. They present methods for overcoming the inherent distortion problems with UWB antenna responses, amplification, and filtering in an indoor multipath environment (Young et al., 2003). Zetik et al. also approached UWB localization using TDOA. They conducted experiments in both the active and passive setting with custom designed SiGe circuit architecture. With their system, they were able to achieve a localization accuracy of within one centimeter (Zetik et al., 2004).

\section{RFID-Based Systems}

Location determination using RFID tags is a difficult problem because tags have extremely limited computational ability to assist the application and a very short reading range. Active tags contain a battery and generally have longer ranges than passive tags. The simplest approach to localizing tags is to use the proximity with readers. The limited reading range can be used to estimate the location of a tag based on the location of a reader (Nara et al., 2005; Philipose et al., 2003).

\section{Experiments with the selected sensor systems}

This section presents the experimental results of the wireless networking technologies (described in Section 2) in different types of building spaces. Advantages and disadvantages of each system are discussed as well. The RFID-based system, however, is excluded in this study because of its short coverage, expensive RFID readers, and the lack of support for pratical applications in a dynamic building environment.

\subsection{Wireless LAN based system}

The accurate positioning of a particular device is a major challenge. State-of-the-art wireless tracking technologies, such as wireless sensors, RFID, and proprietary WLAN basedsensors, typically require a costly dedicated network infrastructure. However, an 802.11 based tracking system can be deployed without significant additional cost. Since many buildings are now rapidly deploying facility-wide 802.11 WLAN infrastructures, a WLANbased tracking approach would provide a cost-effective solution that takes advantages of their wireless infrastructure for asset tracking.

We have deployed a real-time asset visibility system based on an existing 802.11 infrastructure in order to track WLAN tags at the Peter Kiewit Institute (PKI). The system consists of two key software components: a real-time positioning engine that calculates location of assets and a web-based graphical user interface (GUI) that manages system configuration, asset visibility, monitoring and reporting. The positioning engine is based on statistical modeling of received signal strengths and provides accuracy of up to 1.2 meters 
on average. The GUI provides a user friendly web interface that simplifies asset tracking and improves everyday operations, such as asset monitoring and notifications. Since this tracking system is fully software-based, it requires no proprietary network infrastructure.

The first step of the deployment procedure is the data collection phase. Once the access points (APs) were established, the Received Signal Strength (RSS) values were collected from the APs as a function of the mobile's location and orientation. From the measurements, we noted that the RSS values at a given location vary significantly depending on the mobile asset's orientation. Thus, RSS values were collected in each of the four directions (i.e., north, south, east and west) at a number of selected physical locations on the floor. After the data collection phase, the RSS values were imported into the positioning engine and processed to enhance the accuracy of location estimation.

After constructing a database of RSS measurements, called signatures, along with their known 2-dimensional locations and orientations, the system can estimate mobile's position by comparing the measured RSS data to the known signatures in the database. In other words, a mobile device takes a snapshot of RSS from visible APs and compares it with signatures stored in the database. To reduce the computation cost, the search is performed only on some portion of the RSS measurements in the database. If mobile's previous location lies at a point $P$, then the search space is limited to its neighboring points within the distance $d$ from $P$. These neighboring points are grouped into clusters based on their physical closeness. For each cluster, the most probable location of the mobile node is calculated based on the Euclidean distance of RSS measurements. For example, the RSS measurement $\left(p_{1}, p_{2}\right.$, $\left.p_{3}, \ldots, p_{k}\right)$ at a point $P$ and $\left(s_{1}, s_{2}, s_{3}, \ldots, s_{k}\right)$ at point $S$ are the closest if the Euclidean distance between $\mathrm{P}$ and $\mathrm{S}\left(p_{1}-s_{1}\right)^{2}+\left(p_{2}-s_{2}\right)^{2}+\left(p_{3}-s_{3}\right)^{2}+\ldots+\left(p_{k}-s_{k}\right)^{2}$ is minimum. After a number of computations, the system chooses the location with the highest likelihood as the current estimate of the user's location.

\subsubsection{Accuracy}

Under a number of different Wi-Fi network configurations, this study evaluates the accuracy of the system. According to a recommendation from Cisco, a positioning system needs to collect a minimum of three strong and steady Received Signal Strength (RSS) measurements from APs to determine the fine location of Wi-Fi tags with room-level granularity. The Cisco's wireless location appliance guide (Cisco, 2008) also recommends approximately one access point to be placed every 17-20 meters (i.e. roughly one access point is needed every $230-450$ square meter). Thus, in this section, we discuss the accuracy of the system as a function of the number of APs, and the relationship between the AP layout and the location accuracy.

\subsubsection{Impact of the Number of Access Points}

In this study, 8 points on the floor were chosen to measure the Euclidean distance between actual locations and corresponding estimate points. Intuitively, the accuracy would be improved as the number of APs increases. The positions of $10 \mathrm{APs}$ and 8 measurement points are shown in Fig. 1.

The averaged error distance of the deployed system for each point is shown in Tables 1 and 2. As predicted, the error distance gradually decreases as the number of APs increases. This study conducted the same set of experiments with more than 10 APs, but there was no significant improvement in the accuracy of the position estimation. So, the results of the experiments with more than 10 APs were not included. 


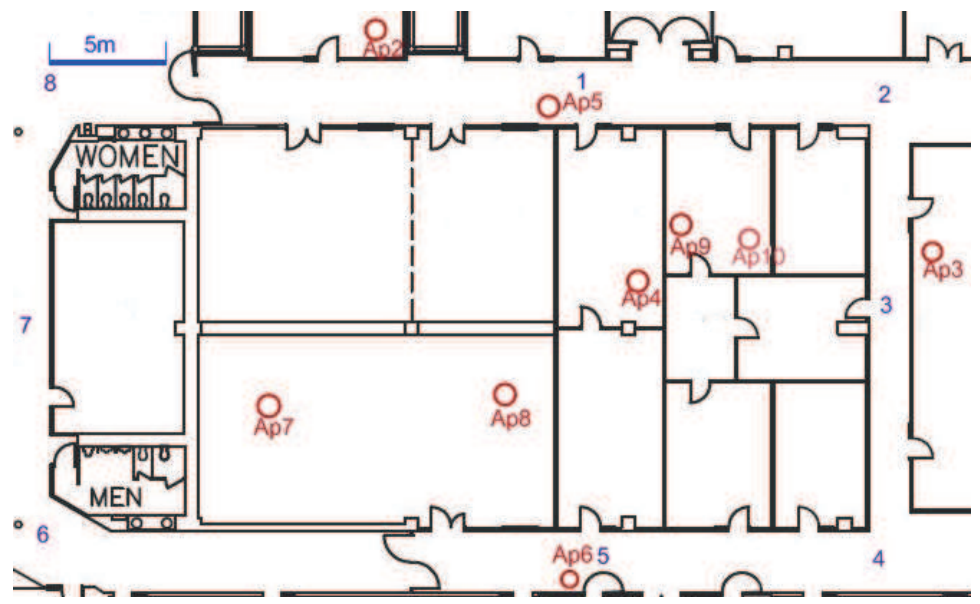

Fig. 1. The Layout of APs

\begin{tabular}{|c|c|c|c|c|}
\hline \multirow{2}{*}{$\begin{array}{c}\text { Measured } \\
\text { Position }\end{array}$} & \multicolumn{4}{|c|}{ Error Distance $(\mathrm{m})$} \\
\cline { 2 - 5 } & $3 \mathrm{APs}$ & 4 APs & 5 APs & 6 APs \\
\hline$\# 1$ & 7.168 & 5.437 & 3.715 & 1.387 \\
\hline$\# 2$ & 3.027 & 2.773 & 0.836 & 1.192 \\
\hline$\# 3$ & 3.522 & 2.72 & 2.931 & 3.125 \\
\hline$\# 4$ & 2.08 & 2.092 & 1.69 & 1.477 \\
\hline$\# 5$ & 5.323 & 4.076 & 3.16 & 3.14 \\
\hline$\# 6$ & 3.971 & 2.611 & 2.351 & 2.294 \\
\hline$\# 7$ & 5.066 & 4.408 & 3.127 & 2.149 \\
\hline$\# 8$ & 0.614 & 0.53 & 0.472 & 0.306 \\
\hline Average & 3.8464 & 3.0809 & 2.2853 & 1.8838 \\
\hline
\end{tabular}

Table 1. Error Distances in Meters (up to 6 APs)

\begin{tabular}{|c|c|c|c|c|}
\hline \multirow{2}{*}{$\begin{array}{c}\text { Measured } \\
\text { Position }\end{array}$} & \multicolumn{4}{|c|}{ Error Distance $(\mathrm{m})$} \\
\cline { 2 - 5 } & $7 \mathrm{APs}$ & $8 \mathrm{APs}$ & $9 \mathrm{APs}$ & $10 \mathrm{APs}$ \\
\hline$\# 1$ & 1.693 & 1.16 & 0.813 & 0.781 \\
\hline$\# 2$ & 2.285 & 1 & 1 & 0.951 \\
\hline$\# 3$ & 1.048 & 2.047 & 1.972 & 1.874 \\
\hline$\# 4$ & 2.543 & 0.873 & 0.784 & 0.774 \\
\hline$\# 5$ & 2.253 & 1.9 & 1.876 & 1.78 \\
\hline$\# 6$ & 1.953 & 1.732 & 1.756 & 1.747 \\
\hline$\# 7$ & 0.245 & 2.5 & 1.396 & 1.207 \\
\hline$\# 8$ & 1.642 & 0.136 & 0.123 & 0.102 \\
\hline Average & 1.693 & 1.4185 & 1.215 & 1.127 \\
\hline
\end{tabular}

Table 2. Error Distances in Meters (up to 10 APs) 


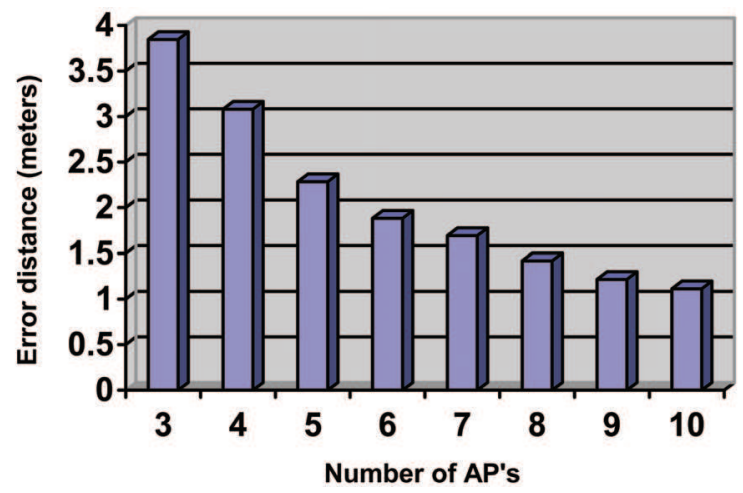

Fig. 2. Impact of the Number of APs on Error Distance

\subsubsection{Impact of the AP Layout}

A Wi-Fi based tracking system provides real-time visibility and tracking. The density and locations of APs are crucial factors in improving the level of accuracy. APs stagger in a way that signals vary in each location. By surrounding the deployment area, the APs provide the greatest chance of achieving room-level positioning. Table 3 shows the error distances for different layouts of the APs. In this experiment, only RSS values from the selected 4 APs were used to estimate the position of Wi-Fi tags. The positions of APs are shown in Fig. 1. As shown in Table 3, the selection of APs has a significant impact on the system accuracy. For example, the accuracy of the last scenario which selects AP\#4, AP\#5, AP\#9, and AP\#10, are poor comparing to the results of other scenarios. This is because, with this AP layout, there are some spots where a tag cannot gather three or more consistent and strong RSS samples from the selected APs.

\begin{tabular}{|c|c|c|c|c|}
\hline \multirow{2}{*}{$\begin{array}{c}\text { Measured } \\
\text { Position }\end{array}$} & \multicolumn{4}{|c|}{ Selected APs } \\
\cline { 2 - 5 } & $1,2,3,4$ & $2,3,6,7$ & $1,2,6,10$ & $4,5,9,10$ \\
\hline$\# 1$ & 5.437 & 0.967 & 1.923 & 2.58 \\
\hline$\# 2$ & 2.773 & 1.992 & 7.302 & 7.323 \\
\hline$\# 3$ & 2.72 & 2.418 & 1.674 & 16.375 \\
\hline$\# 4$ & 2.092 & 1.858 & 2.456 & 2.35 \\
\hline$\# 5$ & 4.076 & 1.525 & 3.282 & 27.817 \\
\hline$\# 6$ & 2.611 & 5.325 & 1.228 & 1.339 \\
\hline$\# 7$ & 4.408 & 2.658 & 2.845 & 4.254 \\
\hline$\# 8$ & 0.53 & 14.965 & 2.565 & 30.369 \\
\hline Average & 3.0809 & 3.9635 & 2.909 & 11.551 \\
\hline
\end{tabular}

Table 3. Error Distance vs. AP Layout (with 4 APs)

\subsubsection{Summary for wireless sensor system}

Due to the extensive Wi-Fi deployments, Wi-Fi tracking is increasingly used in location tracking applications. The Wi-Fi infrastructure enables both customers and developers to quickly develop cost-effective solutions and to create new levels of asset visibility. In most 
Wi-Fi tracking systems, a minimum of three APs must be visible with consistent and strong signal in order to attempt room-level granularity, and actual RF-signature measurements and calibrations are required for finer accuracy. One of the major hurdles of RF-based location tracking is the high overhead associated with collecting RF reference signatures. The RF-signature measurement will be done repeatedly if there is any event that may cause radio signature perturbations, such as varying the number of APs or dislocating the APs.

\subsection{Wireless Sensor Network (WSN) based systems}

Contributing to the field of wireless sensor networks, we focus on indoor tracking systems with wireless sensor motes. We study MoteTrack (Lorincz \& Welsh, 2005), a robust and decentralized RF-based location tracking system developed by a research group at Harvard, and then we added extra features to MoteTrack to address a few issues discussed below. MoteTrack employs a dynamic radio signature distance metric to tolerate failures of the network infrastructure. MoteTrack has two components: beacon motes and mobile motes. The primary function of the beacon motes is to transmit beacon messages at all times (i.e., one beacon message every two seconds). On the other hand, the mobile mote collects the beacon messages from the beacons within its radio range and uses them to estimate its location. Since these tracking systems are radio frequency (RF) based, the location of an object in the given environment is based on the reference signatures collected beforehand. We will describe the differences between MoteTrack and our asset tracking system.

\subsubsection{Differences between MoteTrack and the developed system}

MoteTrack is effective and useful. The location estimation accuracy within 2 meters is in the $80^{\text {th }}$ percentile. MoteTrack operates as follows. During the data collection phase, the beacon motes transmit beacon messages, and the mobile mote collects them and stores the messages on the computer. The collected messages - primarily contain signal strengths, IDs of the beacon motes, and the location $x, y, z$ where the message is collected - are now combined, forming a large database. This large database is then installed on the mobile mote for referencing in the tracking phase. During the location-tracking phase, the beacon motes continue to transmit beacon messages, and the mobile mote captures these messages. Then, a comparison between the current messages and the information from the pre-installed database is done to estimate the location of the mobile mote. The mobile mote, after computing the location $x, y, z$, sends the estimated coordinates to the directly attached computer for displaying.

Mote track is a location support system which allows clients to find out their locations in the building. However, in order to preserve user privacy, the MoteTrack mobile nodes do not report their locations to a centralized server, where a database stores the locations of mobile assets. Although the degree of user privacy offered by MoteTrack could be an important deployment consideration, the location privacy of mobile users trades off many useful services that a tracking system provides. Furthermore, MoteTrack mobile sensors should be directly attached to a display device such as PDA or laptop, and it may not be feasible in many applications due to increased cost and battery issues. Thus, this study modified MoteTrack for tracking mobile assets in a dynamic environment.

In this study, the mobile mote was isolated from a display device. In the new tracking system, there are three components: beacon motes, mobile motes, and a base station. The 
functions of the mobile and beacon motes are essentially the same as in MoteTrack; however, the mobile ones do not have a laptop attached to them in this deployment. Instead, the base station is attached to a tracking server, and it remains at the central location listening to data coming from the mobile motes. The tracking system developed in this study works as follows. The data collection phase is the same as in MoteTrack. Similarly, the large database is installed on the mobile motes for referencing. During the location-tracking phase, the mobile motes collect beacon messages from nearby beacons and then they compute the locations. Then, mobile motes use a multihop routing protocol to route location messages to the base station, using the beacon motes as the intermediate forwarding nodes. Once the location messages arrive at the base station, the data is forwarded to the attached computer or laptop for location display. Fig. 3 shows the differences between the two tracking systems.

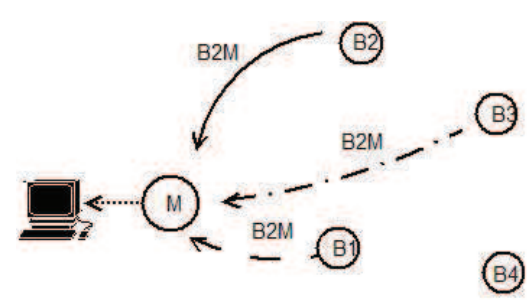

B2M: Message from beacon to mobile motes

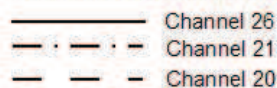

(a) Message Exchange in MoteTrack

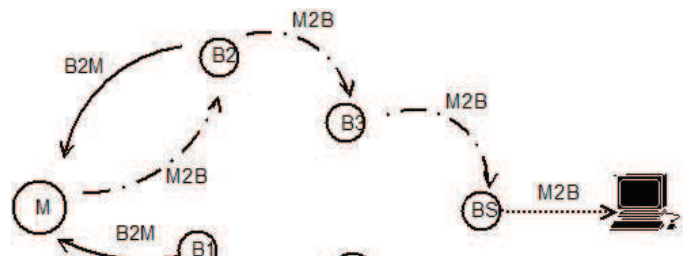

(B4)

B2M: Message from beacon to mobile motes M2B: Message from mobile mote to base station Channel 26

(b) Multihop Message Exchange in Developed Tracking System

Fig. 3. Message Exchanges

\subsubsection{Experimental study and the accuracy of the deployed system}

The test bed was installed at PKI and consists of 20 MicaZ motes, which are indicated as blue dots (labeled from 1 to 20 ) on the building map in Fig. 4 . The red dot (denoted by a 0 ) indicates the base station which collects the position data from the mobile sensor nodes and shows their locations on the GUI. Each mote consists of an 8-bit processor running at $7.3 \mathrm{MHz}$, and it has $128 \mathrm{~KB}$ of read-only program memory, $512 \mathrm{~KB}$ for measurement serial flash, and 4 Kbytes of RAM. The mote operates between $2400 \mathrm{MHz}$ and $2483.5 \mathrm{MHz}$, and it is capable of delivering data up to $250 \mathrm{kbps}$ (Crossbow, Inc., 2008).

Signature collection in Fig. 4 works as follows. The beacon motes (labeled from 1 to 20) send out beacon messages periodically. The user travels along the corridors to collect signatures using a mobile node that is attached to a laptop. Data collection is triggered when the user clicks on the map and creates a numbered mark (e.g., 100, 101, etc); then, the GUI automatically collects the signatures from the mobile node. At this point, the mobile node should receive beacon messages from the beacons. The signature collection phase is done for the entire floor in Fig. 4.. After the collection is completed, the signatures are then processed and are installed onto the mobile node for reference in the position tracking phase. 


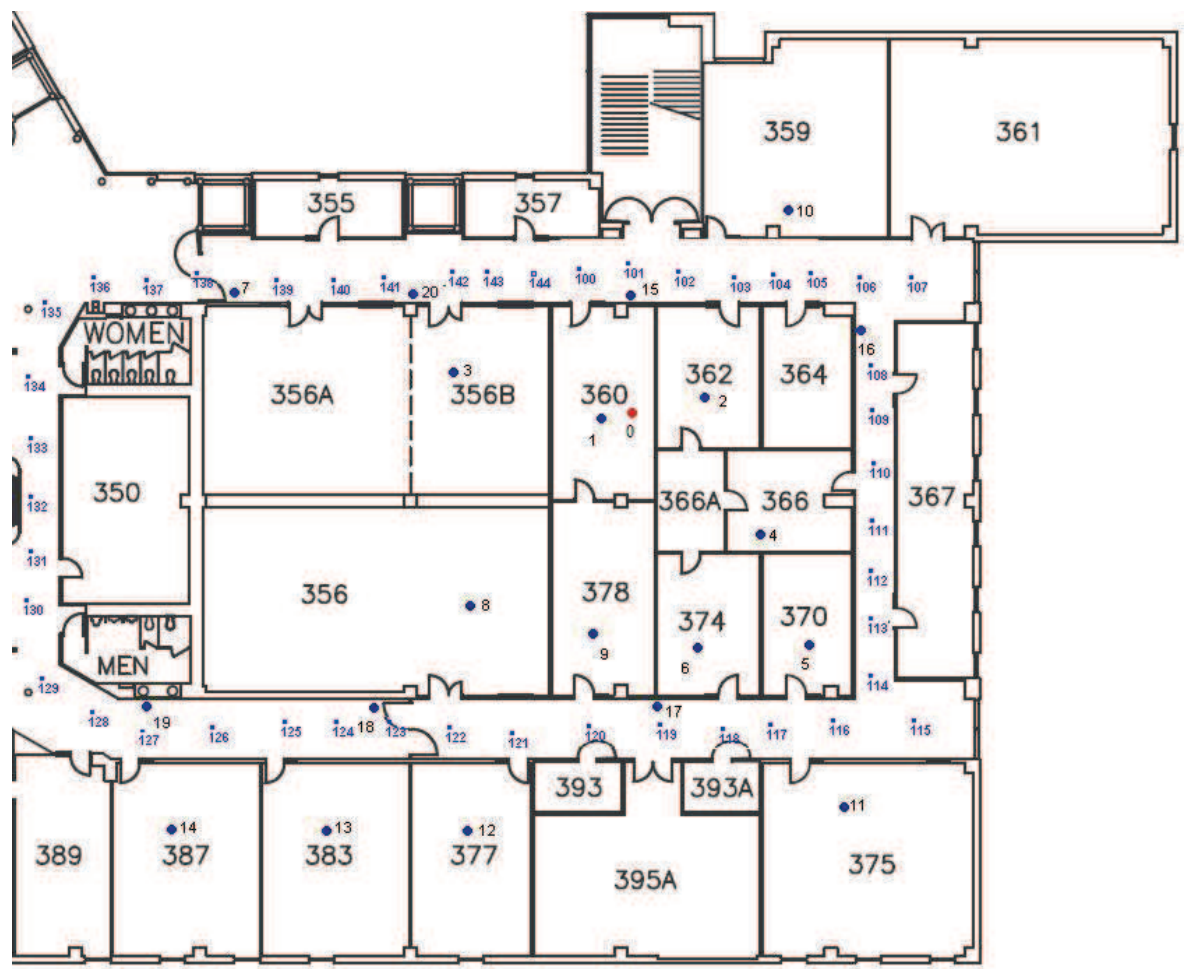

Fig. 4. Deployment of WSN-based Tracking System at the Peter Kiewit Institute (PKI)

\begin{tabular}{|c|c|c|c|c|}
\hline $\begin{array}{c}\text { Error } \\
\text { Distance } \\
\text { (meters) }\end{array}$ & $\begin{array}{c}\text { \# of Samples } \\
\text { (Total 450) }\end{array}$ & $\begin{array}{c}\text { Accumulated } \\
\text { \# of Samples }\end{array}$ & $\begin{array}{c}\text { Percents of } \\
\text { Estimated } \\
\text { Locations }\end{array}$ & $\begin{array}{c}\text { Percentiles of } \\
\text { Estimated } \\
\text { Locations }\end{array}$ \\
\hline $0.0-0.5$ & 139 & 139 & $30.9 \%$ & $30.9 \%$ \\
\hline $0.5-1.0$ & 73 & 212 & $16.2 \%$ & $47.1 \%$ \\
\hline $1.0-1.5$ & 82 & 294 & $18.2 \%$ & $65.3 \%$ \\
\hline $1.5-2.0$ & 71 & 365 & $15.8 \%$ & $81.1 \%$ \\
\hline $2.0-2.5$ & 25 & 390 & $5.6 \%$ & $86.7 \%$ \\
\hline $2.5-3.0$ & 17 & 407 & $3.8 \%$ & $90.5 \%$ \\
\hline $3.0-3.5$ & 10 & 417 & $2.2 \%$ & $92.7 \%$ \\
\hline $3.5-4.0$ & 9 & 426 & $2.0 \%$ & $94.7 \%$ \\
\hline $4.0-4.5$ & 6 & 432 & $1.3 \%$ & $96.0 \%$ \\
\hline $4.5-5.0$ & 8 & 440 & $1.8 \%$ & $97.8 \%$ \\
\hline $5.0-5.5$ & 6 & 446 & $1.3 \%$ & $99.1 \%$ \\
\hline $5.5-6.0$ & 4 & 450 & $.9 \%$ & $100.0 \%$ \\
\hline
\end{tabular}

Table 4. Error Distance in Meters

The accuracy of the system depends on location estimation algorithms, the density of beacon nodes, and the density of reference signatures. The deployed tracking system consists of 20 
beacon nodes distributed across the third floor of PKI. To estimate the accuracy of the system, 45 points on the floor were chosen to measure the Euclidean distance between actual locations and corresponding estimate points. The Euclidean distances were measured 10 times for each point. According to our experimental results, the median resolution of the mote-based RF system is in the range of 1.0 to 1.2 meters, and over $80 \%$ of the location estimates are within 2 meters from their exact position. The results of our experimental study are shown in Table 4 and Fig. 5.

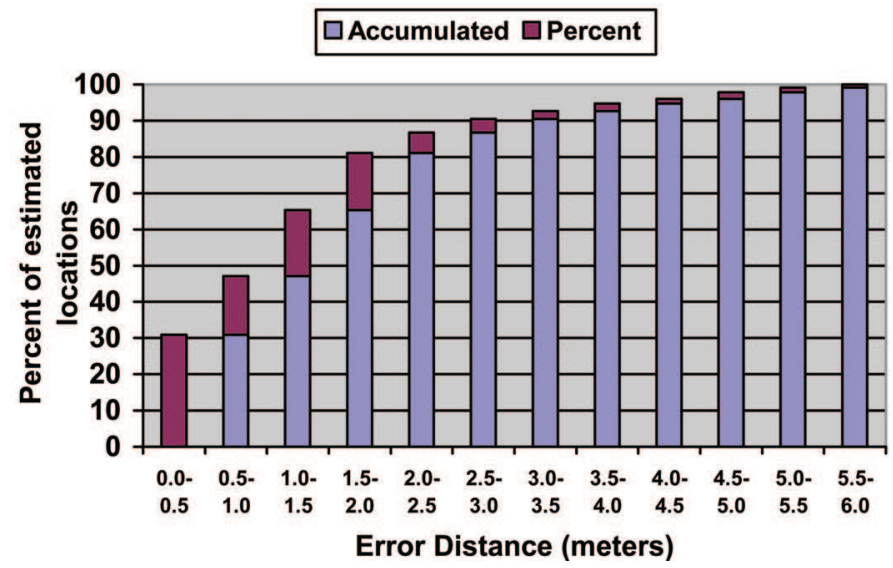

Fig. 5. Error Distance in Percentiles

Since the developed tracking system uses the same methods used in MoteTrack to estimate the location of mobile assets, the accuracy of our system is very similar to that of MoteTrack. For more information on the accuracy of the RF-based sensor tracking system under a wide range of conditions, refer the MoteTrack paper (Lorincz \& Welsh, 2005).

\subsubsection{Summary for wireless sensor system}

MoteTrack is also an RF-based localization system, but it enhances the robustness of the system by employing a distributed approach. According to our experimental study using the WSN-based tracking system deployed at PKI, $80 \%$ of the measured location errors were at or below 2 meters, and the system minimizes the impact of perturbations of the radio signals and provides accurate location estimates with failed beacon nodes. However, the system still has the initial overhead associated with collecting RF reference signatures since the location of a mobile asset is based on the reference signatures collected ahead of time.

\subsection{Ultra-wideband (UWB) systems}

As another indoor position tracking technology, a Federal Communications Commission (FCC) approved, commercial UWB system was tested for implementation and performance in several building spaces. The hardware of the ultra-wideband sensor network consists of tags and sensors. A tag is attached to the object that requires location tracking. As each tag emits an UWB signal, location is calculated using both time difference of arrival(TDOA) between different sensors (a.k.a., receivers) and angle of arrival(AOA) at each sensor. Each sensor employs a minimum of four UWB receivers which allow the angle of arrival to be 
determined (Ubisense 2007). The standard UWB configuration consists of a single master sensor and three slave sensors and requires wired communication cables and timing cables. In order to properly calculate the time of arrival, each slave sensor must synchronize with the master sensor. In the standard configuration, this is done through timing cables between each slave sensor and the master sensor.

While 802.11 systems use standard $2.4 \mathrm{GHz}$ bandwidth, the current UWB system utilizes the 5.8-7.2 GHz bandwidth. While 802.11 uses signal strength, the current UWB system uses TDOA and AOA technologies which enable UWB sensors to have better signal penetration capability and less multi-path signals problems, thus better position accuracy can be obtained.

\subsubsection{Accuracy for UWB}

To evaluate the sensitivity of multi-path signal problems, the accuracy of UWB sensors were tested in an open space and a closed space.

\subsubsection{Open Space Test}

To conduct an open space test, local building's basement was selected. Four sensors are mounted on tripods and multiple known positions are marked on a floor. A total station was used to get the $x, y, z$ position of the sensors and the points on the floor. Fig. 6 shows the surveyed positions of sensors (402-405) and points on the floor (400, 1000-1013). Then a UWB tag was placed on each marked point on the floor to measure the accuracy in difference in distance between a surveyed known position and a wirelessly estimated tag position. The good coverage with four sensors is about $400 \mathrm{~m}^{2}$.

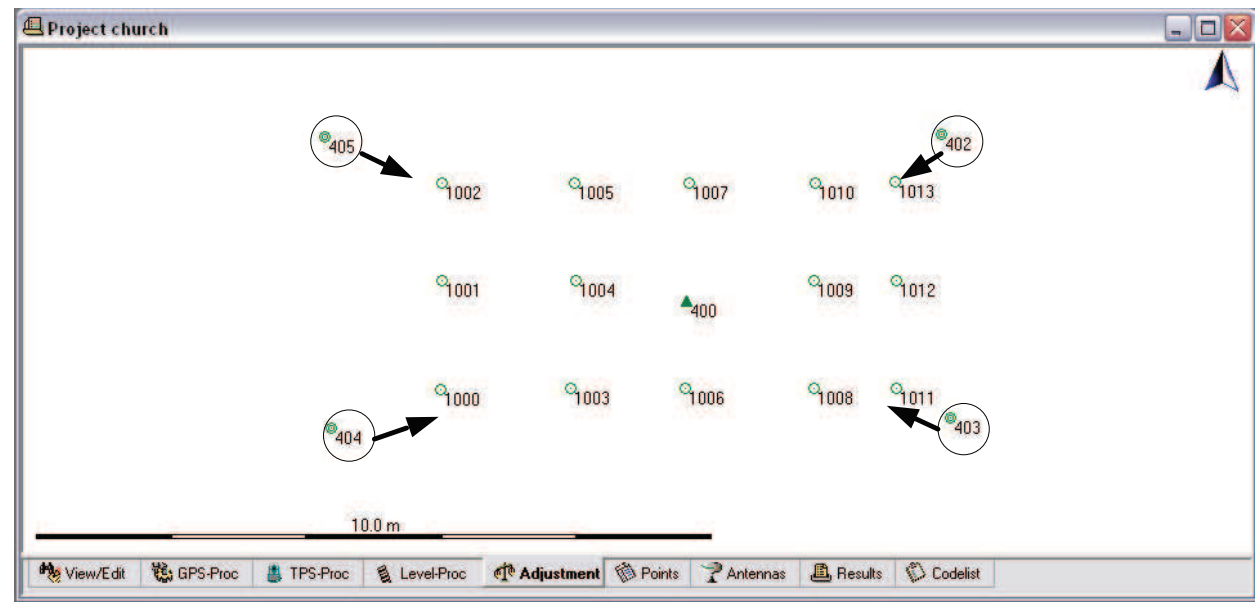

Fig. 6. Test Area Layout

As results, the obtained average accuracy was $19.1 \mathrm{~cm}$. There was a slight accuracy improvement $(1.7 \mathrm{~cm})$ but not significant for most of the points in center $(1001,1004,400,1009,1012)$ when the tag was raised by $35 \mathrm{~cm}$ from the floor level. However, there was a significant accuracy improvement by $13.5 \mathrm{~cm}$ for the outermost points (10001002 and 1011-1013) when the tag was raised by $35 \mathrm{~cm}$. This verifies that in order to accurately determine a tag position, a multiple number of receivers (typically three) must 
have a direct line-of-sight or at most an attenuated line-of-sight transmission path. (Fontana 2003). Especially the receiver did not catch a signal from the tag when the tag is located just below the receiver (See the Fig. 7). Therefore, it is recommended to install the receiver as high as possible from a floor and face it down to cover a large area of the space. Table 5 summarizes the test results.

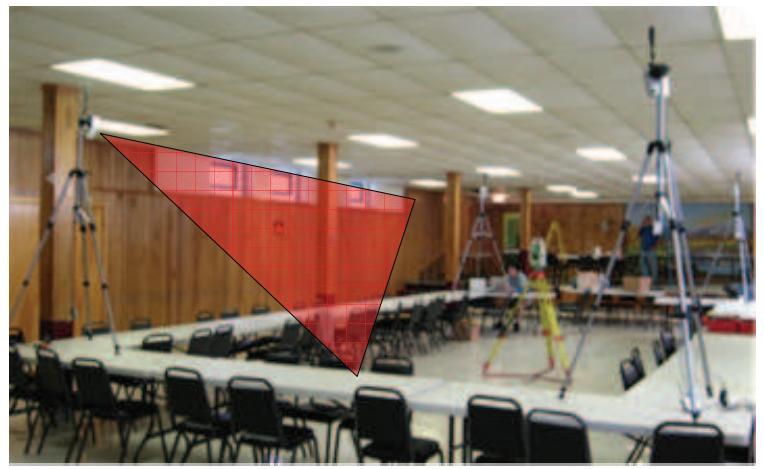

Fig. 7. Open Space Test at a Local Church Basement

\begin{tabular}{|c|c|c|c|c|}
\hline $\begin{array}{c}\text { Overall Average } \\
\text { Accuracy }\end{array}$ & $\begin{array}{c}\text { Average } \\
\text { Accuracy for } \\
\text { Center Points on } \\
\text { Floor Level }\end{array}$ & $\begin{array}{c}\text { Average } \\
\text { Accuracy for } \\
\text { Center Points } \\
\text { raised by } 35 \mathrm{~cm}\end{array}$ & $\begin{array}{c}\text { Average } \\
\text { Accuracy for } \\
\text { Outermost Points } \\
\text { on Floor Level }\end{array}$ & $\begin{array}{c}\text { Average Accuracy } \\
\text { or Outermost Points } \\
\text { raised by } 35 \mathrm{~cm}\end{array}$ \\
\hline $19.1 \mathrm{~cm}$ & $7.9 \mathrm{~cm}$ & $6.2 \mathrm{~cm}$ & $30.0 \mathrm{~cm}$ & $13.8 \mathrm{~cm}$ \\
\hline
\end{tabular}

Table 5. Summary of Test Results

\subsubsection{Closed Space Test}

To conduct a closed space test, an office area of the Peter Kiewit Institute (PKI) was selected. The PKI building was the most challenging subject to test the UWB system because the building (1) was built with steel frames and metal studs, (2) was furnished with all metal furniture and electronics such as printer and copy machines, and (3) had computer electronics and wireless telecommunication labs near by the test area which may cause interferences with the UWB communication system. In addition, heavy people traffic in this area might cause the signal communication error since human bodies can absorb the signal. Especially, $5.8 \mathrm{GHz}$ wireless phones around the testing area should be turned off before testing the UWB system because $5.8 \mathrm{GHz}$ phones were significantly interfered with the current UWB bandwidth, 5.8-7.2 GHz. Fig. 8 shows the test area layout with four receiver's position in 400-403, total station's position in 100-101, and measured tag positions in 500513. The first set of test was conducted by placing a tag on the floor level. Then the tag was raised by $104 \mathrm{~cm}$ for the $2^{\text {nd }}$ test set. Each set of tests was conducted twice. The average of floor level (0 height) tests showed $41 \mathrm{~cm}$ accuracy. When the tag was raised by $104 \mathrm{~cm}$, the test showed $48 \mathrm{~cm}$ accuracy. Unlike the previous open space test which used a stand to raise the tag position, the tag was carried by a human subject in the closed space testbed. Although it was identified that higher position of the tag showed the better accuracy to improve the line-of-sight transmission path, it was noticed in the closed space test that the human carryer significantly affected the accuracy based on its orientation. 


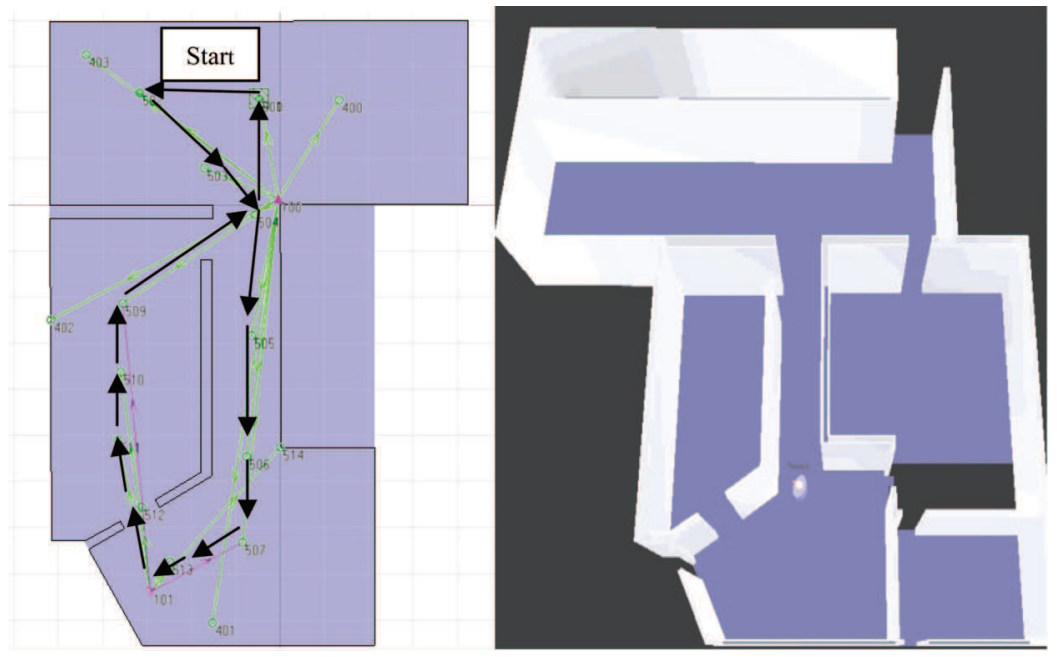

Fig. 8. Closed Space Test Area Layout with Travel Paths (left) and 3D User Interface (right)

\subsubsection{Summary for UWB}

Tag positioning accuracy depends upon several factors, including precise knowledge of all receiver and reference tag locations. Absolute tag position accuracy of better than $19 \mathrm{~cm}$ has been demonstrated in an open space and $48 \mathrm{~cm}$ for the closed space. It is recommended that the receiver need to be located as high as possible to cover larger area. Also, it is important to strategically select direction of receiver and have a minimum of one set of line of sight between a receiver and a tag in any location, which can significantly affect tag's position accuracy.

\section{Implementation of position trackingsystem to mobile robot navigation}

Based on the previous performance test results of the indoor position tracking sensor systems, this study implemented the Ultra-wideband technology to robot's navigation functions mainly due to its higher accuracy than the other two systems. The robot, as shown in Fig. 9, used for this study includes a number of ultrasonic and infrared distance sensors

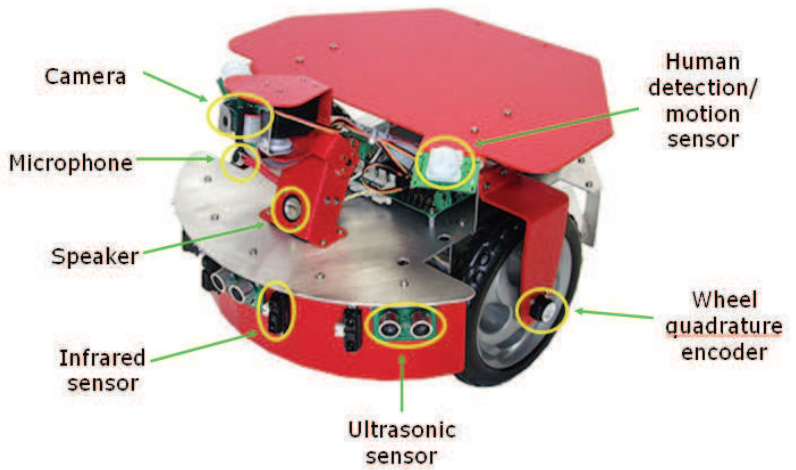

Fig. 9. Wireless Mobile Robot 
aimed to the front, back, and sides, wheel servo sensors, and human detection/motion sensors along with a camera, a microphone, and a speaker (Dr. Robot, 2008). All computations are performed in a centralized manner at a laptop which communicated with the robot wirelessly through an 802.11 channel. Connections are made to each robot through the use of a unique IP address per robot (Fig. 10).

In order to generate location data, a UWB tag was attached to the robot which allowed four UWB sensors to calculate its location.

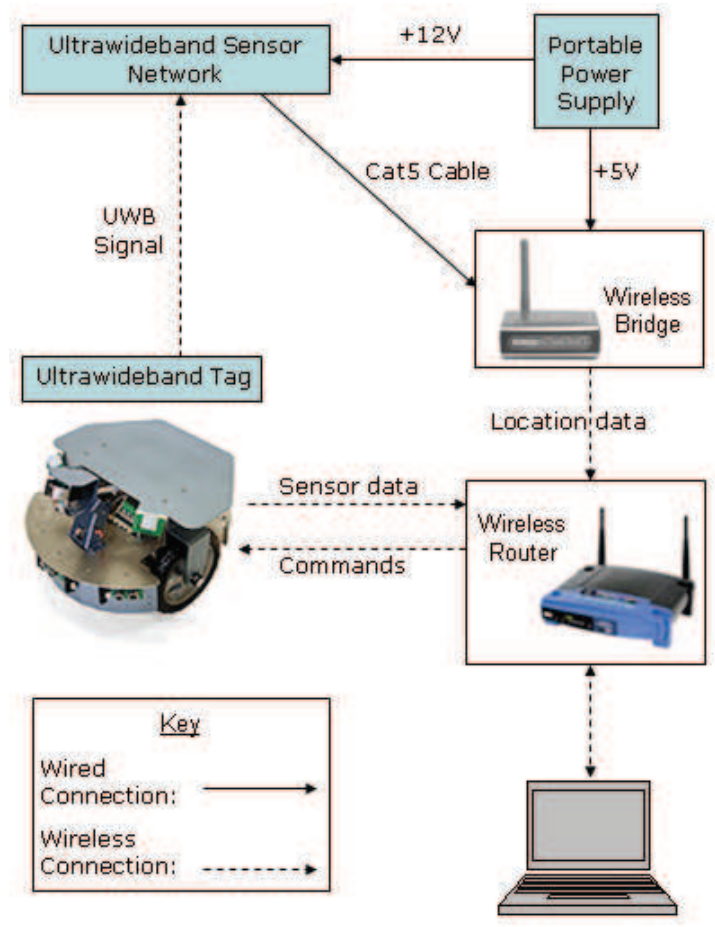

Fig. 10. UWB Integrated Mobile Robot Tracking System Architecture

\subsection{Path planning with ultra-wideband}

The path planning algorithm is divided into two levels. The first is reaction control which handles obstacle avoidance. This level also uses human detection readings to avoid and/or report human presence. If human presence is detected, the robot controller would send an alert to the user and would stop any current movement so the user could intervene and could act appropriately.

The second is end-to-end pathing which determines the route the robot shall follow along the virtual rails from start to end point (see Fig. 11). Here, a well-known routing method, Floyd's all-pairs shortest path algorithm in Fig. 12 (Floyd, 1962), calculates the best path along the rails graph, finds the point closest the robot, and moves to that start point. Then, the robot moves from a point to another along the shortest path until the end point is reached. At this layer, the user may establish a pre-programmed route along the graph where the start and end points are for material delivery or security. 


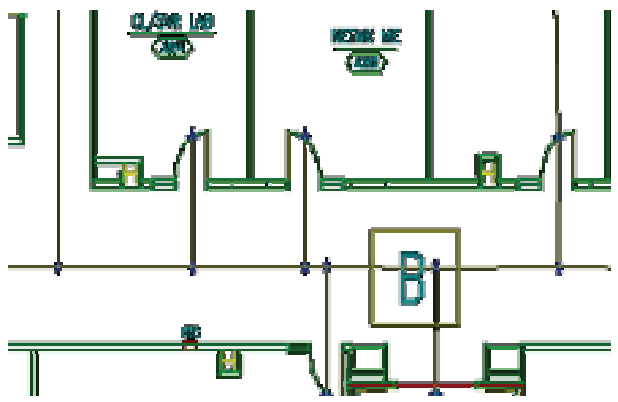

Fig. 11. Rail Graphs in an Office Area

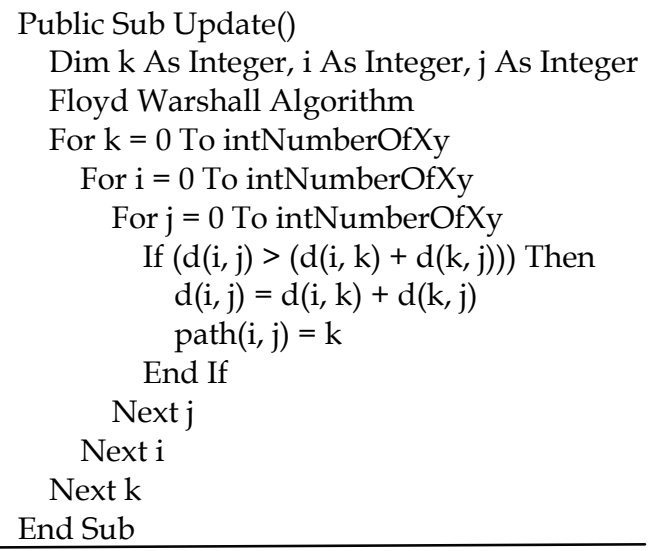

Fig. 12. Floyd's All-pairs Shortest-Path Algorithm coded in Visual Basic

Due to its high accuracy, a UWB tag was mounted on the mobile robot to show robot's current location. UWB sensors provide position data which is sufficiently accurate for the robot navigation. The position data then integrates with Floyd's algorithm to find a shortest path on the rail graphs. A developed filtering model also performs less complex checks such as comparing incoming sensor values against their minimum and maximum defined values to ensure they are within bounds. For example, location data from the UWB sensors are checked using the robot's maximum speed of 1 meter/second as the greatest possible change in position. If an incoming value indicates the robot is traveling more than one meter per second, the value is ignored.

\subsection{Graphical User Interface (GUI)}

In this study, software modules for a robot control and a UWB position tracking have been developed which can be easily imbedded into standard CAD programs such as Microstation and AutoCAD. Since most of the latest building already have either 2D or 3D CAD floor drawings available, the developed modules can save enormous amount of time to generate a new graphical user interface when applied to different building applications. Fig. 13 shows a visual representation of the robot and the environment in which it exists. Location and direction changes are displayed in real time along with human detection alerts. Detected human is also displayed to the user within the drawing when a human detection sensor returns a value indicating human presence. 


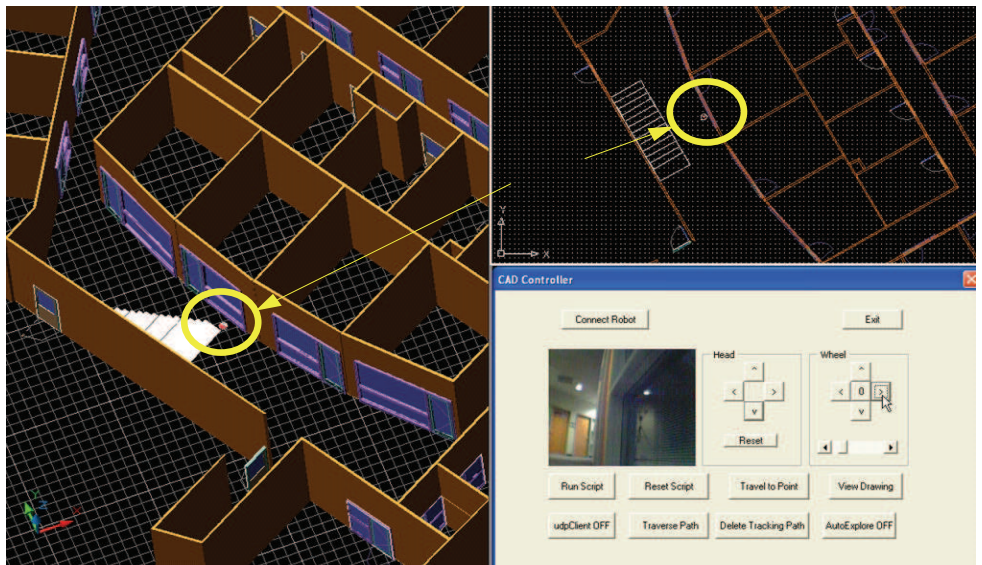

Fig. 13. 3D real-time robot control user interface

\section{Conclusion}

This paper presented an intelligent methodology using wireless position tracking technologies for autonomous mobile robots to improve their navigation functions in dynamic indoor working environments. Three different wireless position tracking technologies were deployed in various types of building spaces, and the accuracy of each system was evaluated. The WLAN based system provides an accuracy of $93 \mathrm{~cm}$ on average when 10 APs are available. For wireless sensor networks, when 20 beacon nodes are available, the median resolution of the mote-based RF system is in the range of 1.0 to 1.2 meters, and over $80 \%$ of the location estimates are within 2 meters. Unlike the other two sensor technologies, the UWB system provides very high accuracies, on average, about $45 \mathrm{~cm}$ with only four receivers (a.k.a. beacons) even in a highly congested office area. Based on the test results of three sensor technologies, this research deploys UWB tag onto a mobile robot to improve the robot's navigation using a well known graphic theory, Floyd's all-pairs shortest path algorithm.

Work continues in order to move away from the rails-based algorithm into a more dynamic method of pathing. In addition, walls and corridors will be taken advantage of to aid in pathing while resolving the fusion of sensor values of sensors with different angles detecting a flat surface displayed in the drawing.

\section{References}

Bahl, P. \& Padmanabhan, V. N. (2000). RADAR: An in-building RF-based User Location and Tracking System, Proceedings of IEEE INFOCOM , pp. 775-784

Battiti, R ; Le Nhat, T. \& Villani, A. (2002). Location Aware Computing: A Neural Network Model for Determining Location in Wireless LANs. Technical Report DIT-02-0083, University of Trento

Cisco. (2008). Cisco 2700 Series Wireless Location Appliance Deployment Guide. http:// www.cisco.com/univercd/cc/td/doc/product/wireless/loc2700/12700/depgd.pdf

Crossbow, Inc. (2008). http:/ / www.xbow.com/, 2008

Dr. Robot. Inc. (2008). www.drrobot.com, May 1, 2008

Ekahau. (2008) http://www.ekahau.com, 2008 
Floyd, Robert W. (1962). Algorithm 97: Shortest Path. Communications of the ACM, Vol. 5 No. 6, p. 345

Fontana, R.; Richley, E. \& Barney, J. (2003). Commercialization of an Ultra Wideband Precision Asset Location System, IEEE conference on Ultra Wideband Systems and Technologies, Reston, VA, November 2003

Gezici, S.; Zhi Tian; Giannakis, G.B.; Kobayashi, H.; Molisch, A.F.; Poor, H.V. \& Sahinoglu, Z. (2005). Localization Via Ultra-wideband Radios: A Look at Positioning Aspects for Future Sensor Networks. IEEE Signal Processing Magazine, Vol. 22, No. 4, pp. 70- 84

Haeberlen, A.; Flannery, E.; Ladd, A. M.; Rudys, A.; Wallach, D. S. \& Kavraki, L. E. (2004). Practical Robust Localization Over Large-scale 802.11 Wireless Networks, Proceedings of the 10th Annual International Conference on Mobile Computing and Networking, pp. 70-84, 2004, ACM Press, New York, NY, USA

Harter, A.; Hopper, A; Steggles, P.; Ward, A. \& Webster, P. (1999). The Anatomy of a Context-Aware Application, Proceedings of $5^{\text {th }}$ International Conference on Mobile Computing and Networking, pp.59-68, ACM Press, Seattle, WA

Hightower, J. \& Borriello, G. (2001). Locating Systems for Ubiquitous Computing. IEEE Computer, Vol. 34, No. 8, 2001, pp.57-66

Kohn, L.; Corrigan, J. \& Donaldson, M. (2000). To Err Is Human: Building a Safer Health System, Committee on Quality of Health Care in America, Institute of Medicine

Ladd, A. M.; Bekris, K. E.; Rudys, A.; Kavraki, L. E.; Marceau, G. \& Wallach, D. S. (2002). Roboticsbased Location Sensing Using Wireless Ethernet, Proceedings of the 8th Annual International Conference on Mobile Computing and Networking (MOBICOM), September 2002

Liu, X.; Corner, M. \& Shenoy, P. (2006). Ferret: RFID Localization for Pervasive Multimedia, Proceedings of the 8th Ubicomp Conference, September 2006

Lorincz, K. \& Welsh, M. (2005). Motetrack: A robust, Decentralized Approach to RF-based Location Tracking, Proceedings of the International Workshop on Location and ContextAwareness (LoCA 2005), May 2005.

Nara, T.; Onoda, H.; Yamane, J. \& Ando S. (2005). Dipole Estimation from the Magnetic Field Gradient for RFID Tag Localization. Transactions of the Society of Instrument and Control Engineers, Vol. 1, No. 1, pp. 16-20, 2005

Pango networks. (2008). http://www.pangonetworks.com, 2008

Philipose, M.; Fishkin, K.; Fox, D.; Hahnel, D. \& Burgard, W. (2003). Mapping and Localization with RFID Technology. Technical Report IRS-TR-03-014, Intel Corporation, December 2003

Priyantha, N. B.; Chakraborty, A. \& Balakrishnan, H. (2000). The Cricket Location-Support System, Proceedings of the 6th Annual International Conference on Mobile Computing and Networking, pp. 32-43, 2000, ACM Press, New York, NY, USA

Skibniewski, M. \& Jang, W. (2007). Localization Technique for Automated Tracking of Construciton Materials Utilizing Combined RF and Ultrasound Sensor Interfaces, Proceedings of the 2007 International Workshop on Computing in Civil Engineering, pp. 657-664, Pittsburgh, PA, July 2007

Ubisense. (2007). www.ubisense.net, November 30, 2007

Want, R.; Hopper, A.; Falcao V. \& Gibbons, J. (1992). The Active Badge Location System. ACM Transactions on Information Systems, Vol. 10, pp. 91-102, January 1992

Young, D.P.; Keller, C.M.; Bliss, D.W. \& Forsythe, K.W. (2003). Ultra-wideband (UWB) Transmitter Location Using Time Difference of Arrival (TDOA) Techniques. Conference Record of the Thirty-Seventh Asilomar Conference on Signals, Systems and Computers, Vol. 2, pp. 1225-1229, November 2003

Zetik, R.; Sachs, J. \& Thoma R. (2004). UWB Localization-Active and Passive Approach, Proceedings of the 21st IEEE Instrumentation and Measurement Technology Conference, IMTC 04., Vol. 2, pp. 1005-1009, May 2004 


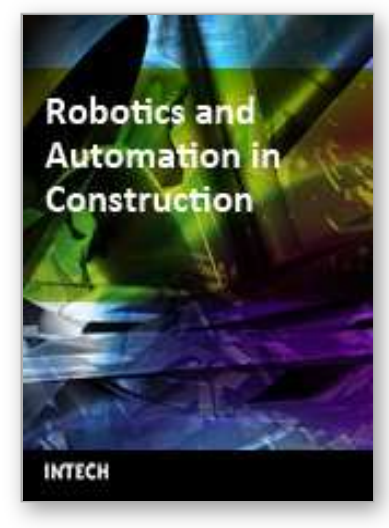

\author{
Robotics and Automation in Construction \\ Edited by Carlos Balaguer and Mohamed Abderrahim
}

ISBN 978-953-7619-13-8

Hard cover, 404 pages

Publisher InTech

Published online 01, October, 2008

Published in print edition October, 2008

This book addresses several issues related to the introduction of automaton and robotics in the construction industry in a collection of 23 chapters. The chapters are grouped in 3 main sections according to the theme or the type of technology they treat. Section I is dedicated to describe and analyse the main research challenges of Robotics and Automation in Construction (RAC). The second section consists of 12 chapters and is dedicated to the technologies and new developments employed to automate processes in the construction industry. Among these we have examples of ICT technologies used for purposes such as construction visualisation systems, added value management systems, construction materials and elements tracking using multiple IDs devices. This section also deals with Sensorial Systems and software used in the construction to improve the performances of machines such as cranes, and in improving Human-Machine Interfaces (MMI). Authors adopted Mixed and Augmented Reality in the MMI to ease the construction operations. Section III is dedicated to describe case studies of RAC and comprises 8 chapters. Among the eight chapters the section presents a robotic excavator and a semi-automated façade cleaning system. The section also presents work dedicated to enhancing the force of the workers in construction through the use of Robotic-powered exoskeletons and body joint-adapted assistive units, which allow the handling of greater loads.

\title{
How to reference
}

In order to correctly reference this scholarly work, feel free to copy and paste the following:

Yong K. Cho, Jong-Hoon Youn and Nam Pham (2008). Performance Tests for Wireless Real-Time Localization Systems to Improve Mobile Robot Navigation in Various Indoor Environments, Robotics and Automation in Construction, Carlos Balaguer and Mohamed Abderrahim (Ed.), ISBN: 978-953-7619-13-8, InTech, Available from:

http://www.intechopen.com/books/robotics_and_automation_in_construction/performance_tests_for_wireless_ real-time_localization_systems_to_improve_mobile_robot_navigation_in_

\section{INTECH}

open science | open minds

\section{InTech Europe}

University Campus STeP Ri

Slavka Krautzeka 83/A

51000 Rijeka, Croatia

Phone: +385 (51) 770447

\section{InTech China}

Unit 405, Office Block, Hotel Equatorial Shanghai

No.65, Yan An Road (West), Shanghai, 200040, China

中国上海市延安西路65号上海国际贵都大饭店办公楼 405 单元

Phone: +86-21-62489820 
Fax: +385 (51) 686166

Fax: +86-21-62489821

www.intechopen.com 
(C) 2008 The Author(s). Licensee IntechOpen. This chapter is distributed under the terms of the Creative Commons Attribution-NonCommercialShareAlike-3.0 License, which permits use, distribution and reproduction for non-commercial purposes, provided the original is properly cited and derivative works building on this content are distributed under the same license. 